Article

\title{
High-Performance Ni-Co Sulfide Nanosheet-Nanotubes Grown on Ni Foam as a Binder Free Electrode for Supercapacitors
}

\author{
Jaffer Saddique, Xiaopeng Cheng, Huifeng Shi, Rui Wu and Yuefei Zhang * \\ Institute of Microstructure and Property of Advanced Materials, Beijing University of Technology, \\ Beijing 100124, China \\ * Correspondence: yfzhang@bjut.edu.cn
}

Received: 23 May 2019; Accepted: 22 July 2019; Published: 31 July 2019

\begin{abstract}
The novel hierarchical Ni-Co sulfide nanosheet-nanotubes arrays were directly grown on $\mathrm{Ni}$ foam, as binder-free electrodes, have been successfully synthesized following a one-step facile hydrothermal method combined with a sulfide treatment. The initial value of the area capacitance achieved $2.28 \mathrm{~F} \mathrm{~cm}^{-2}$ at a current density of $1 \mathrm{~mA} \mathrm{~cm}^{-2}$. A high areal capacitance retention of $95.2 \%$ compared to activation-induced peak value is achieved after 3000 charge-discharge cycles, which is much better than counter Ni-Co oxide electrode $\left(1.75 \mathrm{~F} \mathrm{~cm}^{-2}\right.$ at $1 \mathrm{~mA} \mathrm{~cm}{ }^{-2}, 93.2 \%$ retention compared to activation induced peak value). The outstanding and excellent super capacitive performance is ascribed to ion-exchange reaction, which induces a flexible hollow nanotube feature and show higher conductivity, compared with Ni-Co oxide NWs. Cyclic voltammetry (CV) and Electrochemical impedance spectra (EIS) results confirmed that the synthesized electrode contains the lowest resistance at high, and at lower frequency, leading to easy penetration of electrolytes and fast transportation of electrons inside the electrode. In this proposed work, a one-step hydrothermal method has been followed, and provided for the sulfide-induced, with a noticeable electrochemical performance of nickel cobaltite compounds and supplying a promising route for high-performance supercapacitor electrodes.
\end{abstract}

Keywords: Ni-Co sulfide; nanotubes; nickel foam; cation exchange; supercapacitors

\section{Introduction}

In recent years, sustainable energy and green energy systems get considerable attention to meet the increasing demand for energy throughout the world. Much effort has been invested to extend renewable energy sources [1,2], for example, rechargeable batteries and supercapacitors [3-5] are the main focus for research and get considerable attraction as energy-storage devices. Recently, supercapacitor received considerable attention, due to some of its interesting and beneficial advantages, like a long life time, high power density, and fast charge-discharge process. The electrochemical classification of a supercapacitor is based on charge-discharge progress and also on energy storage mechanisms, which can be classified into two types: Electrochemical double layer capacitors and pseudo-capacitors: (1) The electrochemical double layer capacitors can store energy directly by ion adsorption at the interface of the electrode/electrolyte and the main electrodes are carbon based material; (2) the pseudo-capacitors use the redox-active materials i.e., metal oxide-based material and conductive polymers, as electrode material allow the insertion redox reaction for both surface, as well at bulk state, and through fast surface redox reactions for storing energy [6,7].

The performance of supercapacitors is mainly connected with the properties of the electrode material. Generally, three types of electrode materials are being used in a supercapacitor: Carbonaceous 
materials, transition metal oxides, and conducting polymer materials. Carbon-based materials, such as the typical materials of double-layer capacitors have a relatively lower specific capacitance and lower energy density, which hinders its practical application. In contrast, the metal oxides, as pseudo-capacitance materials, preserving much higher capacitance owing to the more charge storage at surface, get great interest and are widely investigated, due to their high theoretical capacitance for supercapacitors. The fabrication of these compounds, and especially oxides materials with diverse structures and morphologies, have attracted significant interest in material science and also at an industry level. Recently, efforts have also been made in the synthesis of binary component materials to upgrade the characteristics of materials and to enhance their characteristic properties beyond each individual component. Therefore, tremendous efforts have been devoted to the synthesis and fabrication of transition metal oxides, such as $\mathrm{NiO}$ [8-10], $\mathrm{Fe}_{2} \mathrm{O}_{3}$ [11,12], $\mathrm{MnO}_{2}$ [13-15], $\mathrm{Co}_{3} \mathrm{O}_{4}$ [16-19], due to their high energy density, low cost, and environmental friendliness. Recently, cobalt sulfides and nickel sulfides, as new transitional metal sulfide electrode materials with high electrical conductivity and with superior electrochemical performances, have been investigated for pseudocapacitors [20,21]. However, the chemical and physical properties of transition metal sulfides are directly related to surface morphology, structure, and the size of the materials. The fabrication of these compounds, and especially sulfides material similar to oxides materials with diverse structures and morphologies, have attracted significant interest for developing electrode materials for energy storage devices [22]. Up until now, different types of structures, with a diverse morphology of $\mathrm{Ni} / \mathrm{Co}$ sulfide, such as nanosheets [22], porous aerogels [23], urchin-like structures [24], have been reported. More importantly, the nanotube feature structures shows promising electrochemical performances with an increased rate of capability, as they provide an easy way for electrolyte penetration into active electrode material from the inner nanotube surface and in reducing the inactive electroactive surface area. As a result, a more electroactive surface area of electrode materials contribute to the electrochemical performances of the supercapacitors [25]. Additionally, in the comparison Ni-Co sulfide nanostructure, supported on conductive substrates, shows excellent pseudo-capacitive performances relatively to the nanostructure powders, because there is a chance that ion diffusion and electron transport are more quickly blocked during electrochemical performances when introducing the binders to make electrodes [26].

Based on the above considerations, in this paper, we adopted a one-step hydrothermal method, combined with thermal treatment, to synthesize Ni-Co sulfides nanotubes on $\mathrm{Ni}$ foam as a binder-free electrode for pseudocapacitors. The fabricated Ni-Co sulfides electrode on nickel foam possess a superior electrochemical performance (initial value is $2.28 \mathrm{~F} \mathrm{~cm}^{-2}$ ) to the pristine $\mathrm{NiCo}_{2} \mathrm{O}_{4}$ pattern (the initial value is $1.75 \mathrm{~F} \mathrm{~cm}^{-2}$ ) at current density of $1 \mathrm{~mA} \mathrm{~cm}^{-2}$. Furthermore, a high areal capacitance $2.20 \mathrm{~F} \mathrm{~cm}^{-2}$ with $95.2 \%$ retention, compared to the activation-induced peak value is achieved at $1 \mathrm{~mA} \mathrm{~cm}^{-2}$ current density is achieved after 3000 charge-discharge cycles, which is much better than counter $\mathrm{Ni}$-Co oxide electrode (a high areal capacitance $1.91 \mathrm{~F} \mathrm{~cm}^{-2}$ with $93.2 \%$ retention compared to activation induced peak value). Herein, we adopted the hydrothermal method combined with thermal treatment for the synthesis of $\mathrm{Ni}$-Co sulfide compounds to improve the capacitance. Our results indicate that the Ni-Co sulfide nanosheet-nanotubes arrays can act as high-performance electrode materials in supercapacitors.

\section{Experimental Section}

\subsection{The Synthesis of $\mathrm{NiCO}_{2} \mathrm{O}_{4}$ Nanosheet-Nanowire Arrays}

A one-step facile hydrothermal method was used for the fabrication of Cobalt Nickle Oxide nanoarrays on Ni foam combined with post thermal treatment. During the experiment the used reagents were of analytical grade and kept away from any further purification. To remove NiO layer, $2 \mathrm{M} \mathrm{HCl}$ was used carefully to clean the $\mathrm{Ni}$ foam, and then deionized water and absolute ethanol were used to wash the $\mathrm{Ni}$ foam -for $20 \mathrm{~min}$ respectively. Cobalt nitrate $\left(\mathrm{Co}\left(\mathrm{NO}_{3}\right)_{2} \cdot 6 \mathrm{H}_{2} \mathrm{O}\right)$, Nickelous nitrate $\left(\mathrm{Co}\left(\mathrm{NO}_{3}\right)_{2} \cdot 6 \mathrm{H}_{2} \mathrm{O}\right)$, ammonium fluoride $\left(\mathrm{NH}_{4} \mathrm{~F}\right)$, and urea were obtained from Beijing Chemical Reagent 
Company. In a typical way, $0.38 \mathrm{~g}$ of $\mathrm{Co}\left(\mathrm{NO}_{3}\right)_{2} \cdot 6 \mathrm{H}_{2} \mathrm{O}, 0.19 \mathrm{~g}$ of $\mathrm{Ni}\left(\mathrm{NO}_{3}\right)_{2} \cdot 6 \mathrm{H}_{2} \mathrm{O}$ and $1.0 \mathrm{~g}$ of urea, $0.37 \mathrm{~g}$ of $\mathrm{NH}_{4} \mathrm{~F}$ were dissolved in $40 \mathrm{~mL}$ of deionized water and was subject to continuous magnetic stirring until a homogenous pink solution was achieved. Then the $\mathrm{Ni}$ foam $\left(1 \times 2 \mathrm{~cm}^{2}\right.$ in area) and the aqueous solution were transferred to $50 \mathrm{~mL}$ Teflon-lined stainless steel autoclave where the Ni foam of about $1 \times 1 \mathrm{~cm}^{2}$ in area was protected from solution contamination by uniformly coating the top with a polytetrafluoroethylene tape) and maintained at $120^{\circ} \mathrm{C}$ for $6 \mathrm{~h}$. Afterwards, a red thin product was obtained on $\mathrm{Ni}$ foam when cooled down to room temperature and subsequently washed with deionized water and ethanol for several time, dried at $80^{\circ} \mathrm{C}$ for $6 \mathrm{~h}$, and then finally calcined at $300^{\circ} \mathrm{C}$ in air for $2 \mathrm{~h}$.

\subsection{The Synthesis of Ni-Co Sulfide Nanosheet-Nanotube Arrays}

The Ni-Co sulfide nanosheet-nanotube arrays were obtained through a hydrothermal method by treating the as-obtained $\mathrm{NiCo}_{2} \mathrm{O}_{4}$ nanosheet-nanowire arrays with sodium sulphide $\left(\mathrm{Na}_{2} \mathrm{~S}\right)$. In detail, $1.20 \mathrm{~g} \mathrm{Na} 2 \mathrm{~S}_{2} \cdot 9 \mathrm{H}_{2} \mathrm{O}$ was first dissolved in $40 \mathrm{~mL}$ deionized water and transferred into $50 \mathrm{~mL}$ Teflon container with the $\mathrm{NiCo}_{2} \mathrm{O}_{4}$ sample. Then the autoclave was sealed and kept in an oven at $120{ }^{\circ} \mathrm{C}$. After $12 \mathrm{~h}$, the autoclave was carried out and cooled down to the room temperature. Then, the product on $\mathrm{Ni}$ foam was obtained and subsequently washed with deionized water before being dried at $80^{\circ} \mathrm{C}$ for $6 \mathrm{~h}$.

\subsection{Structural Characterization}

The crystalline structure of the synthesized Ni-Co nano-arrays nanotube were examined by X-ray diffraction (XRD; Bruker D8) with Cu Ka radiation $(\lambda=0.154 \mathrm{~nm})$. The state valence were characterized through X-ray photoelectron spectroscopy (XPS; Thermo Scientific Escalab 250Xi). The surface morphological studied were carried out by scanning electron microscopy (SEM; FEI Quanta 250) and transmission electron microscopy (TEM; JEOL, JEM-2010), elemental distribution were examined by energy-dispersive $\mathrm{X}$-ray spectroscopy (EDS) system, while high resolution-transmission electron microscope (HRTEM; JEOL, JEM-2010 F) is for the investigation of lattice structure. The EDS mapping experiments were conducted by transmission electron microscopy (TEM, FEI Titan G2, operated at $300 \mathrm{kV}$ ). Areal capacitances were calculated using Equation (1) [27].

$$
\mathrm{Ca}=\mathrm{I} \times \Delta \mathrm{t} /(\mathrm{S} \times \Delta \mathrm{V})
$$

where constant discharge current were measured as $\mathrm{I}(\mathrm{mA}), \Delta \mathrm{t}(\mathrm{s})$ is the total discharge time, $\Delta \mathrm{V}(\mathrm{v})$ is the potential drop during discharge and $\mathrm{S}$ is the electrochemical active area of the electrode.

\subsection{Electrochemical Tests}

A typical way is adopted for measuring the electrochemical performance and properties of the working electrode using three-electrode mode cells, prepared with a working electrode, while a platinum foil is used as the counter electrode, $\mathrm{Hg} / \mathrm{HgO}$ is used as the reference electrode, and $6 \mathrm{M}$ $\mathrm{KOH}$ aqueous solution is used as electrolyte. The electrochemical characteristics cyclic voltammetry $(\mathrm{CV})$ were tested in the fixed potential window ranging between 0 and $0.6 \mathrm{~V}$ at different scan rates while that of electrochemical impedance spectroscopy (EIS) with the Ni-Co sulfides-Ni foam composite $\left(1 \mathrm{~cm}^{2}\right.$ area $)$ as the working electrode were measured at open circuit in the frequency range from $0.01 \mathrm{~Hz}$ to $100 \mathrm{kHz}$ while applying potential $5 \mathrm{mV}$ were carried out at CHI660D (Chenhua, Shanghai) work station., The Galvanostatic charge-discharge (GCD) of $\mathrm{NiCo}_{2} \mathrm{O}_{4}$ and the corresponding Ni-Co sulfide nickel foam were performed at $1 \mathrm{mV} \mathrm{s}^{-1}$ and $1 \mathrm{~mA} \mathrm{~cm}{ }^{-2}$ and the cycle measurements both were performed on a LAND battery test system (LAND, Wuhan). 


\section{Results}

The two-step environmental growth scheme of the Ni-Co sulfide nanosheet-nanotube on nickel foam is illustrated in Figure 1. Firstly, the $\mathrm{NiCO}_{2} \mathrm{O}_{4}$ precursor was firstly grown directly on Ni foam via following facile hydrothermal method, which is one of the most cost effective, environmentally friendly, and easy approachable non-toxic scientific methods for synthesizing controllable different morphology. Then, we carried out a calcination treatment so that the $\mathrm{NiCo}_{2} \mathrm{O}_{4}$ nanosheet-nanowire arrays on $\mathrm{Ni}$ foam can be converted hydrothermally to Ni-Co sulfide nanosheet-nanotube arrays counterparts by reacting with $\mathrm{Na}_{2} \mathrm{~S}$, which is from attribution to the anion-exchange reaction between $\mathrm{O}^{2-}$ and $\mathrm{S}^{2-}$.

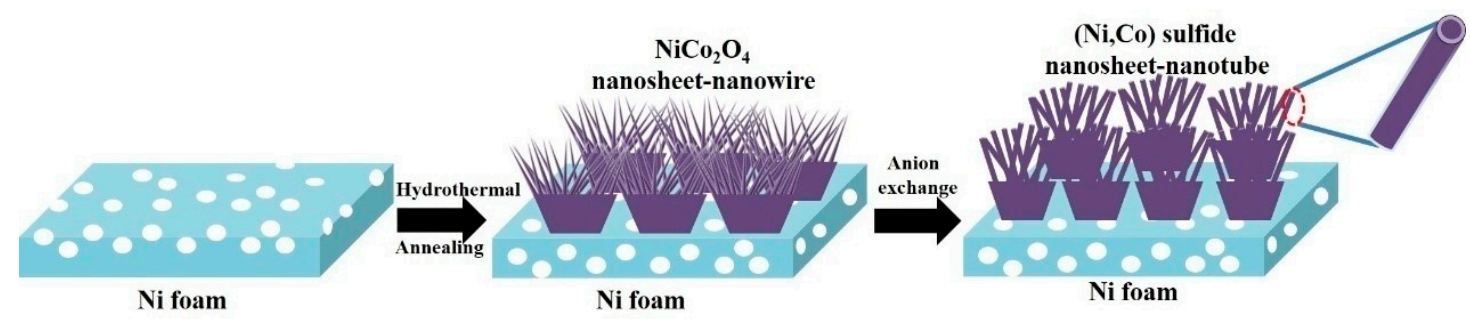

Figure 1. Schematic illustration of the synthesis processes of Ni-Co sulfide nanosheet-nanotube arrays.

Figure 2 presents the XRD pattern of the successful preparation of Ni-Co sulfide. The pure $\mathrm{NiCo}_{2} \mathrm{O}_{4}$ phase is observed in the pristine pattern, while after sulfurizing (anion-exchange) process, the final products can be indexed to mixture of $\mathrm{Co}_{4} \mathrm{~S}_{3}$ (JCPDS No. 42-1448, $\mathrm{a}=\mathrm{b}=\mathrm{c}=9.387$ ) and $\mathrm{Ni}_{3} \mathrm{~S}_{2}$ (JCPDS No. 44-1418, $\mathrm{a}=\mathrm{b}=5.745, \mathrm{c}=7.135$ ), in which $\mathrm{Co}_{4} \mathrm{~S}_{3}$ can be readily indexed to the (311), (400), (422), (511) and (440) and $\mathrm{Ni}_{3} \mathrm{~S}_{2}$ can be indexed to the(101), (110), (021), (211), (213), (122), and (300). There are no other diffraction peaks, implying the full conversion of the precursor into $\mathrm{Ni}$-Co sulfide during the hydrothermal process. XPS was performed to further confirm the prepared sample, while the well fitted spectra of $\mathrm{Ni}, \mathrm{Co}, \mathrm{S}$ (Figure S1) illustrates the successful anion-exchange reaction between $\mathrm{O}^{2-}$ and $\mathrm{S}^{2-}$. The Ni $2 \mathrm{p}$ and $\mathrm{Co} 2 \mathrm{p}$ spectra of Ni-Co sulfide (Figure S1a,b) are well fitted into $\mathrm{Ni}^{2+}, \mathrm{Ni}^{3+}, \mathrm{Co}^{2+}$, and $\mathrm{Co}^{3+}$ accompanied with shakeup satellite, respectively. While, $\mathrm{S} 2 \mathrm{p}$ spectra (Figure S1c) display strong peaks at $161.6 \mathrm{eV}$ and $163 \mathrm{eV}$, which can be associated with $\mathrm{S}^{2-}$ species in the composite and sulfur ions in low coordination at the surface [28].

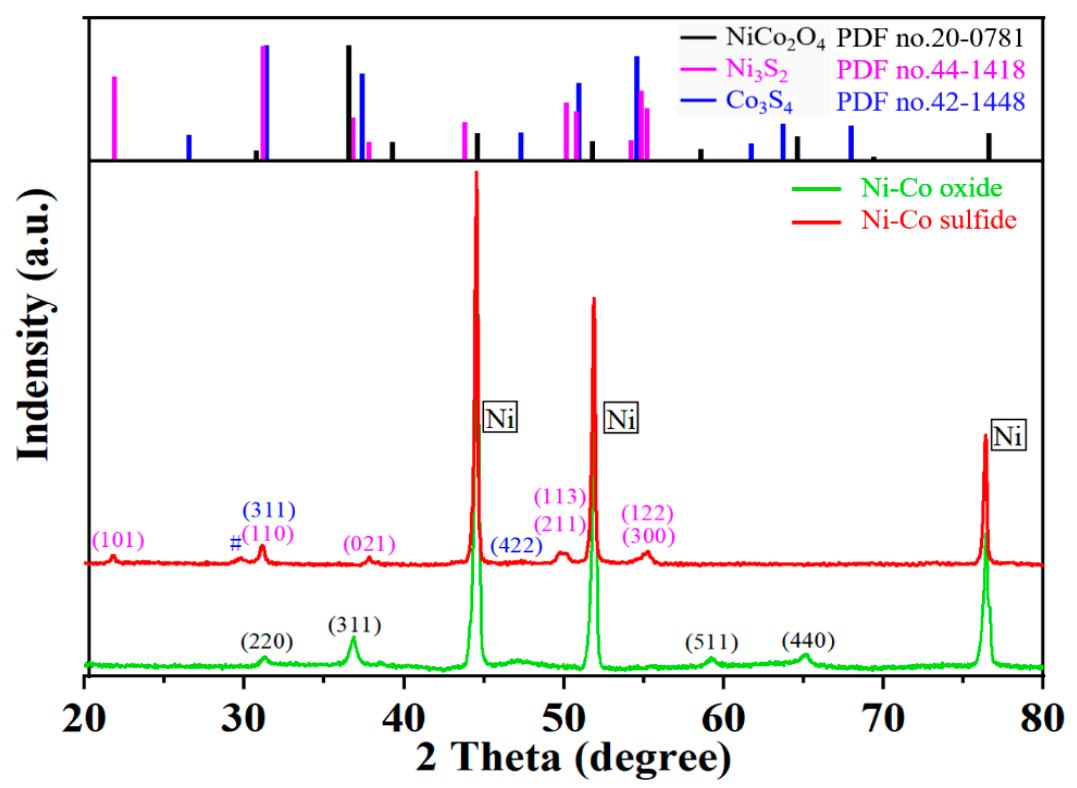

Figure 2. XRD pattern of as prepared Ni-Co sulfide arrays supported on Ni foam. 
To better elucidate the morphology, representative SEM images were carried out from the samples are given in Figure 3. The morphology of pristine Ni-Co oxide structures shown in Figure 3a,b demonstrates that nickel foam substrate surface was uniformly covered with a layer of uniform active material. The magnified SEM images (Figure 3c,d) show that large-scale, high dense and aligned $\mathrm{Ni}-\mathrm{Co}$ sulfide arrays are supported on nickel foam substrate. EDS mapping of $\mathrm{Ni}$-Co oxide and $\mathrm{Ni}-\mathrm{Co}$ sulfide as shown in Figure S2. To demonstrate the morphology characters of Ni-Co sulfide arrays on $\mathrm{Ni}$ foam, the sulfide pattern was treated by focus ion beam irradiation. In the Figure 3e, the nanotubes in left part of the sample are wiped out by the Ga ion irradiation and the nanosheets feature is clearly exposed out of the arrays. And obviously the nanotubes (blue arrow) still left are connected with the nanosheet (yellow arrow), proving the formation of nanosheet-nanotubes feature of Ni-Co sulfide.
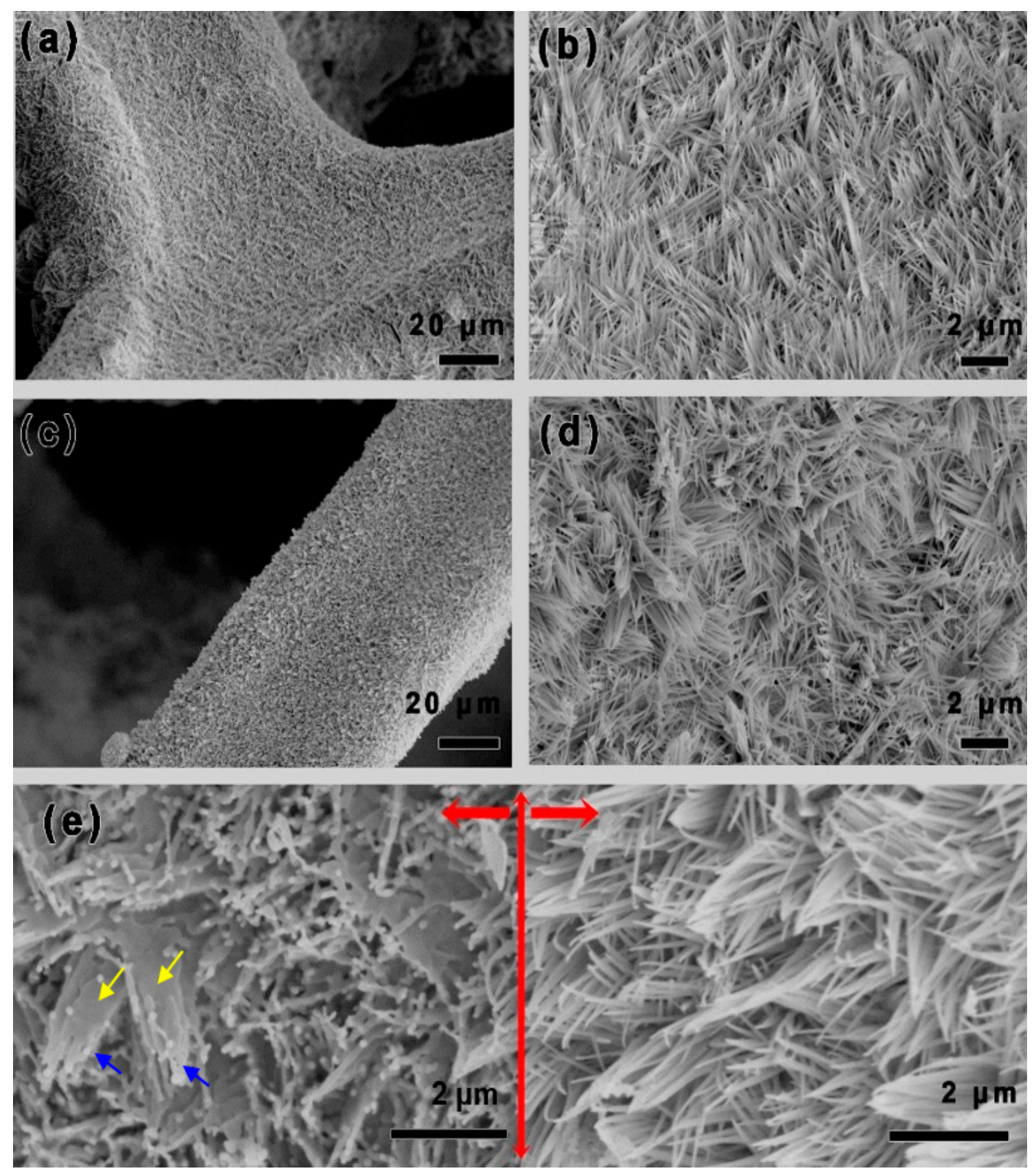

Figure 3. SEM images of (a,b) Ni-Co oxide, (c,d) Ni-Co sulfide, and (e) the Ni-Co sulfide partially treated by focus ion beam irradiation. 
The closely structural information and morphology of the Ni-Co sulfide were investigated by TEM in Figure 4. A full view of a single hierarchical porous Ni-Co sulfide nanosheet-nanotube array in Figure 4a apparently shows the array consists of nanosheet below and nanotubes epitaxial growth from the nanosheet. The SAED pattern in the Figure $4 \mathrm{~b}$ demonstrates the polycrystalline nature of the $\mathrm{Ni}-\mathrm{Co}$ sulfide nanowire, the well-defined diffraction rings can be readily indexed to the $\mathrm{Co}_{3} \mathrm{~S}_{4}(220)$, (222), (400), (422), (511), (440), (800), and $\mathrm{Ni}_{3} \mathrm{~S}_{2}$ (021), (202), (211), (122), respectively. Furthermore, the magnified TEM images in Figure 4c-e show that the Ni-Co sulfide nanowires are hollow internally with a sheath thickness of $\sim 15 \mathrm{~nm}$ (Figure $4 \mathrm{~d}$ ). The nanotube and rough surface are very both effective in increasing the electroactive sites. The lattice fringes of HTTEM image in Figure $4 \mathrm{f}$ and $\mathrm{g}$ can be assigned to the (220) and (220) crystal plane of $\mathrm{Co}_{3} \mathrm{~S}_{4},(201)$, and (202) plane of $\mathrm{Ni}_{3} \mathrm{~S}_{2}$, respectively. The sample contains the elements of $\mathrm{Co}, \mathrm{Ni}$, and $\mathrm{S}$ evidenced by EDS pattern in Figure $4 \mathrm{~h}$, demonstrating that it is completely in sulfuration.

To demonstrate the electrochemical performance and properties of $\mathrm{Ni}-\mathrm{Co}$ sulfide nanosheet-nanotube arrays, the comparative experiment was carried out between the $\mathrm{NiCo}_{2} \mathrm{O}_{4}$ electrode and the Ni-Co sulfide electrode are shown and plotted in Figure 5. Firstly, a comparison of the cyclic voltammetry (CV) and galvanostatic charge-discharge (GCD) curves at $1 \mathrm{mV} \mathrm{s}^{-1}$ and $1 \mathrm{~mA} \mathrm{~cm}^{-2}$ of $\mathrm{NiCO}_{2} \mathrm{O}_{4}$ and the corresponding Ni-Co sulfide nickel foam are shown in Figure 5a,b. Conspicuously, the integral area of the $\mathrm{CV}$ curve and the discharge time of Ni-Co sulfide is larger than $\mathrm{Ni}$-Co oxides, implying excellent electrochemical energy storage capacity of the synthesized Ni-Co sulfide nanotube. Figure $5 \mathrm{c}$ shows the $\mathrm{CV}$ curves of Ni-Co sulfide at different scan rates from 1 to $10 \mathrm{mV} \mathrm{s}^{-1}$ ranging in a potential window between " 0 to $0.6 \mathrm{~V}$ ". The pair redox peaks can clearly be observed and visible in all the CV curves leading to different pseudo-capacitive characteristics, during the electrochemical performance test and are directly related to the Faradaic redox reactions to $\mathrm{M}-\mathrm{O} / \mathrm{M}-\mathrm{O}-\mathrm{OH}$ [29], where $\mathrm{M}=\mathrm{Ni}$ or Co. In Figure 5c, as the scan rate increases, the redox current increases; in the result, the corresponding anodic/cathodic peaks shifted towards higher and lower potential specifically at $8 \mathrm{mV} \mathrm{s}^{-1}$, and at $10 \mathrm{mV} \mathrm{s}^{-1}$, respectively with the large potential separation. This phenomenon is caused by the kinetic irreversibility in the redox process, due to Ohmic resistance and polarization behavior of the electrode [30]. The galvanostatic charge discharge curves of Ni-Co sulfide electrode with. $\mathrm{Hg} / \mathrm{HgO}$ reference electrode in the potential window ranging between 0 to $0.5 \mathrm{~V}$ at different selected current densities changing from 1 to $8 \mathrm{~mA} \mathrm{~cm}^{-2}$, as shown in Figure $5 \mathrm{~d}$. It can clearly observe that distinct plateau regions of the Ni-Co sulfide galvanostatic charge discharge are matching with the redox peaks in CV curves, indicates high pseudocapacitive characteristics of the $\mathrm{Ni}$-Co sulfide. The cycle stability of supercapacitors for long-term is another critical issue to further evaluate the application of different structure electrodes. The cycling tests for the four synthesized electrodes were carried out by the repeated charging-discharging measurement at current density of $1 \mathrm{~mA} \mathrm{~cm}^{-2}$ over 3000 cycles. The long cycle performance and stability at a current density of $1 \mathrm{~mA} \mathrm{~cm}^{-2}$ are shown in Figure 5e, the Ni-Co sulfide nanotube achieved their peak $\left(2.31 \mathrm{~F} \mathrm{~cm}^{-2}\right)$ after 30 cycles and then became quite stable with $95.2 \%$ of the retained specific capacitance after 3000 cycles, while, the counter-part $\mathrm{NiCo}_{2} \mathrm{O}_{4}$ nanowire array exhibited activation induced peak $\left(2.05 \mathrm{~F} \mathrm{~cm}^{-2}\right)$, which is attributed the enhanced conductivity and surface sites [31], then, followed by an obvious capacity decline to $1.91 \mathrm{~F} \mathrm{~cm}^{-2}$ after 3000 cycles. We can see that the cycling performance of Ni-Co oxide supercapacitor increases at the beginning and then decreases during the charging/discharging measurements. This is because the electrode cannot be completely wetted at the pristine cycles and has to go through a slow activation process for the electrode [32]. Furthermore, compared with previous work, as shown in Table S1, we clearly see that the Ni-Co sulfide nanosheet-nanotubes is a kind of excellent electrode material for supercapacitors. 

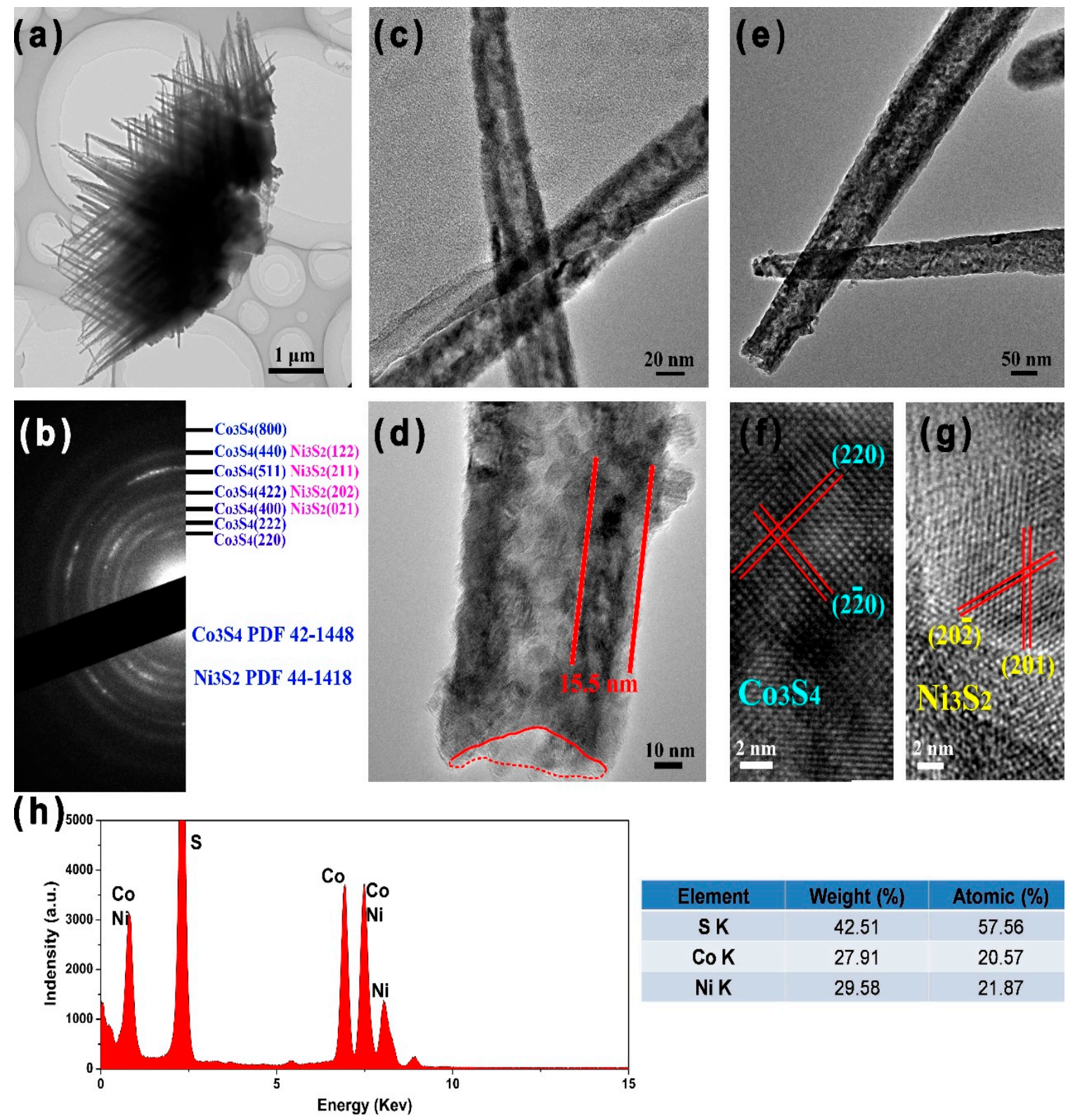

Figure 4. (a) TEM image of single Ni-Co sulfide nanosheet-nanotube arrays scratched off the $\mathrm{Ni}$ foam and (b) corresponding Selected area electron diffraction (SAED) pattern, (c-e) Magnified TEM images, showing the hollow structure. (f,g) Corresponding HRTEM image, (h) Energy-dispersive X-ray spectroscopy (EDS) pattern of the sulfide nanosheet-nanotube nanoarrays.

Furthermore, electrochemical impedance spectroscopy (EIS) test were measured to evaluate the electrochemical capacitance properties and stability of the Ni-Co sulfide electrode and is illustrated in Figure $5 \mathrm{f}$, the tests were measured at open circuit in the frequency range from $0.01 \mathrm{~Hz}$ to $100 \mathrm{kHz}$ while applying potential applying $5 \mathrm{mV}$. The obtained Nyquist plots of the Ni-Co oxide and sulfide electrodes contain semicircles part in high frequency and a linear part at low frequency region. The diameter of the semicircle and the straight short line of the obtained EIS spectra represent the total resistance during charge-transfer, the interfacial of the electrolyte resistance, and the resistance of the interface of the active material/current collector during electrochemical reaction. EIS result indicates the enhancement in the ion diffusion and effective electron transfer in the electrode charge-discharge process after sulfide, resulting in the excellent comprehensive electrochemical performance compared to Ni-Co oxide electrode. 
(a)

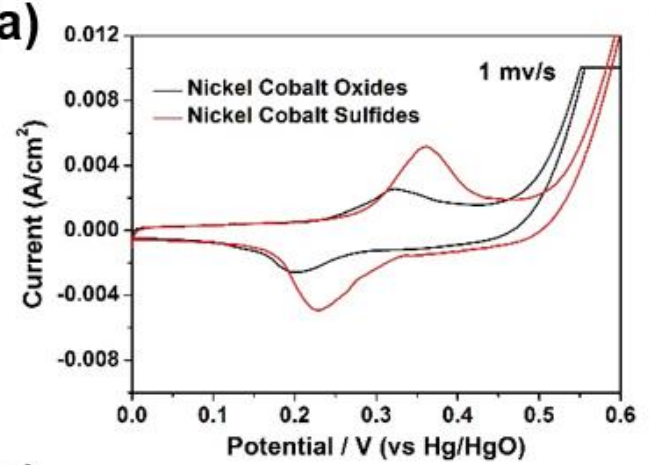

(c)

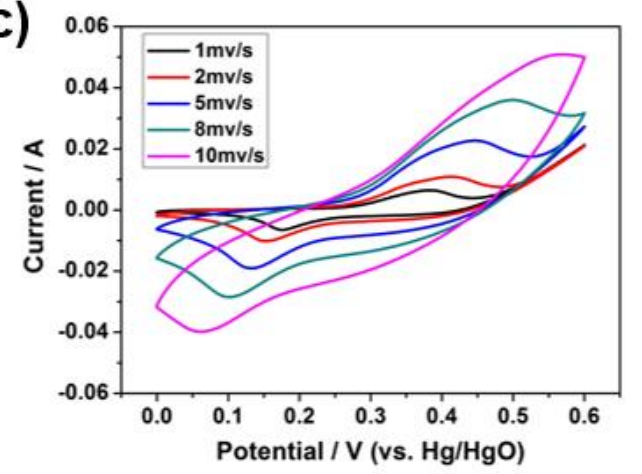

(e)

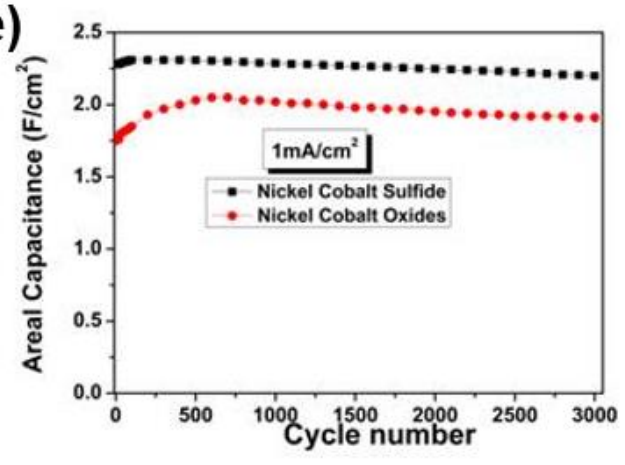

(b)

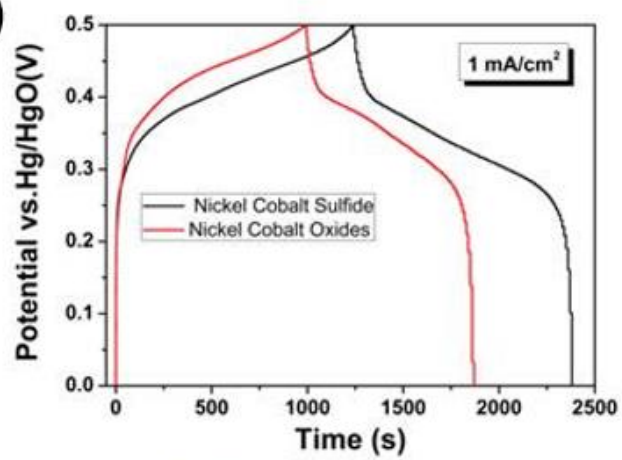

(d)

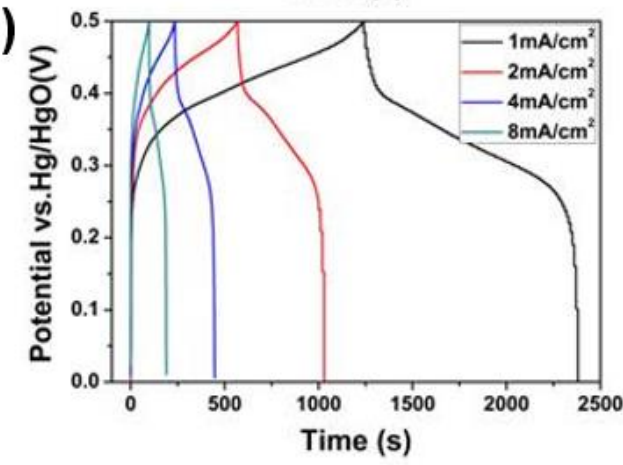

(f)

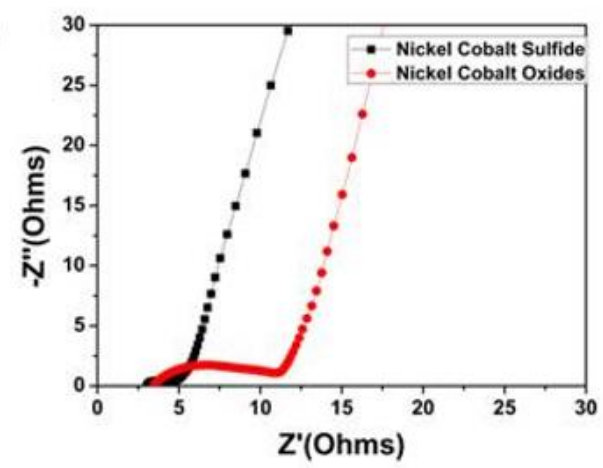

Figure 5. Electrochemical performance of Ni-Co sulfide electrode and the pristine $\mathrm{NiCo}_{2} \mathrm{O}_{4}$ electrode. The electrochemical measurements were carried out in a $6 \mathrm{M} \mathrm{KOH}$ aqueous solution as electrolyte. (a) Cyclic voltammetry (CV) curves comparison, (b) Galvanostatic charge-discharge curves, (c) CV curve of Ni-Co sulfide electrode, (d) Galvanostatic charge-discharge measurements of Ni-Co sulfide electrode, (e) cycle performance comparison and (f) EIS curve comparison.

\section{Conclusions}

In summary, the novel growth of Ni-Co sulfide nanosheet-nanotube arrays as a binder free electrode on $\mathrm{Ni}$ foam have been successfully synthesized for supercapacitors, based on the anion-exchange reaction, by utilizing a one-step facile hydrothermal method combined with a subsequent low-cost sulfuration. The electrochemical performances of the transition metal oxides and sulfide electrodes materials are directly dependent on the shape, size, and morphological features obtained during the experimental process. The initial value of the area capacitance achieved $2.28 \mathrm{~F} \mathrm{~cm}^{-2}$ of the present electrode at a current density of $1 \mathrm{~mA} \mathrm{~cm}^{-2}$, a high areal capacitance retention of $95.2 \%$ is achieved compared to the activation induced peak value even after 3000 charge-discharge cycles, which is much better than counter $\mathrm{Ni}-\mathrm{Co}$ oxide electrode $\left(1.75 \mathrm{~F} \mathrm{~cm}^{-2}\right.$ at $1 \mathrm{~mA} \mathrm{~cm}{ }^{-2}$, 93.2\% retention after 3000 charge-discharge cycles compared to activation induced peak value). The excellent super-capacitive performance is ascribed to the ion-exchange reaction, which induces the flexible hollow nanotube feature and higher conductivity, compared with Ni-Co oxide NWs. 
These results evidently suggest the potential of the Ni-Co sulfide nanocomposite electrode for high-performance supercapacitors.

Supplementary Materials: The following are available online at http:/www.mdpi.com/2076-3417/9/15/3082/s1, Figure S1: The XPS spectra of Ni-Co sulfide. (a) Ni 2p, (b) Co 2p, (c) S 2p, Figure S2: STEM-HAADF image and its corresponding EDS maps. (a-d) The sample of $\mathrm{NiCo}_{2} \mathrm{O}_{4}$, (e-h) the sample of Ni-Co sulfide. Table S1: Reference survey of related active material electrodes for electrochemical capacitors.

Author Contributions: J.S. and X.C. both contributed equally. Methodology and experiment, J.S., X.C. and R.W.; Writing-original draft preparation, X.C. and J.S.; writing-review and editing, H.S.; funding acquisition and supervision, Y.Z.

Funding: This work was supported by funding from the International Research Cooperation Seed Fund of Beijing University of Technology (2018A18), the NSFC (21676005), the NSFCDFG joint project (51761135129), Beijing Municipal Natural Science Foundation (2172002), Great Wall Scholarship Project (CIT and TCD20170306).

Conflicts of Interest: The authors declare no conflict of interest.

\section{References}

1. Armand, M.; Tarascon, J.M. Building better batteries. Nature 2008, 451, 652-657. [CrossRef] [PubMed]

2. Wang, L.; Chen, B.; Ma, J.; Cui, G.; Chen, L. Reviving lithium cobalt oxide-based lithium secondary batteries-toward a higher energy density. Chem. Soc. Rev. 2018, 47, 6505-6602. [CrossRef] [PubMed]

3. Miller, J.R.; Simon, P. Electrochemical capacitors for energy management. Science 2008, 321, 651-652. [CrossRef] [PubMed]

4. Fic, K.; Platek, A.; Piwek, J.; Frackowiak, E. Sustainable materials for electrochemical capacitors. Mater. Today 2018, 21, 437-454. [CrossRef]

5. Zhu, Y.; Murali, S.; Stoller, M.D.; Ganesh, K.J.; Cai, W.; Ferreira, P.J. Carbon-Based Supercapacitors Produced by Activation of Graphene. Science 2011, 332, 1537-1541. [CrossRef]

6. Nguyen, T.; Montemor, M.D.F. Metal Oxide and Hydroxide-Based Aqueous Supercapacitors: From Charge Storage Mechanisms and Functional Electrode Engineering to Need-Tailored Devices. Adv. Sci. 2019, 6, 1801797. [CrossRef] [PubMed]

7. Nguyen, T.; Montemor, M.F. Redox view active materials for metal compound based hybrid electrochemical energy storage: A perspective. Appl. Surf. Sci. 2017, 422, 492-497. [CrossRef]

8. Cai, G.; Wang, X.; Cui, M.; Darmawan, P.; Wang, J.X.; Lee-Sie Eh, A.; Lee, P.S. Electrochromo-supercapacitor based on direct growth of $\mathrm{NiO}$ nanoparticles. Nano Energy 2014, 12, 258-267. [CrossRef]

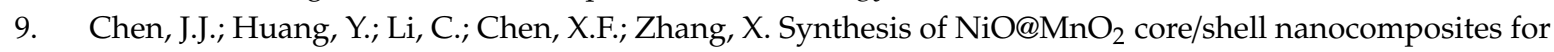
supercapacitor application. Appl. Surf. Sci. 2016, 360, 534-539. [CrossRef]

10. Yang, Y.; Xia, X.; Ye, H.; Wang, L.; Jiao, X.; Lei, W.; Hao, Q. Three-Dimensional Hierarchical Structure $\mathrm{ZnO@C@NiO} \mathrm{on} \mathrm{Carbon} \mathrm{Cloth} \mathrm{for} \mathrm{Asymmetric} \mathrm{Supercapacitor} \mathrm{with} \mathrm{Enhanced} \mathrm{Cycle} \mathrm{Stability.} \mathrm{ACS} \mathrm{Appl.}$ Mater. Interfaces 2018, 10, 3549-3561. [CrossRef]

11. Zhao, J.; Li, Z.; Yuan, X.; Yang, Z.; Zhang, M.; Meng, A.; Li, Q. A High-Energy Density Asymmetric Supercapacitor Based on $\mathrm{Fe}_{2} \mathrm{O}_{3}$ Nanoneedle Arrays and $\mathrm{NiCo}_{2} \mathrm{O}_{4} / \mathrm{Ni}(\mathrm{OH})_{2}$ Hybrid Nanosheet Arrays Grown on SiC Nanowire Networks as Free-Standing Advanced Electrodes. Adv. Energy Mater. 2018, 8, 1702787. [CrossRef]

12. Rakhi, R.B.; Chen, W.; Cha, D.; Alshareef, H.N. Substrate Dependent Self-Organization of Mesoporous Cobalt Oxide Nanowires with Remarkable Pseudocapacitance. Nano Lett. 2012, 12, 2559-2567. [CrossRef] [PubMed]

13. Yuan, C.; Yang, L.; Hou, L.; Shen, L.; Zhang, X.; Lou, X.W. Growth of ultrathin mesoporous $\mathrm{Co}_{3} \mathrm{O}_{4}$ nanosheet arrays on $\mathrm{Ni}$ foam for high-performance electrochemical capacitors. Energy Environ. Sci. 2012, 5, 7883-7887. [CrossRef]

14. Ma, W.; Nan, H.; Gu, Z.; Geng, B.; Zhang, X. Superior performance asymmetric supercapacitors based on $\mathrm{ZnCo}_{2} \mathrm{O}_{4} @ \mathrm{MnO}_{2}$ core-shell electrode. J. Mater. Chem. A 2015, 3, 5442-5448. [CrossRef]

15. He, Y.; Chen, W.; Li, X.; Zhang, Z.; Fu, J.; Zhao, C.; Xie, E. Freestanding Three-Dimensional Graphene/ $\mathrm{MnO}_{2}$ Composite Networks As Ultralight and Flexible Supercapacitor Electrodes. ACS Nano 2013, 7, 174-182. [CrossRef] [PubMed]

16. Liu, T.; Zhang, L.; You, W.; Yu, J. Core-Shell Nitrogen-Doped Carbon Hollow Spheres $/ \mathrm{Co}_{3} \mathrm{O}_{4}$ Nanosheets as Advanced Electrode for High-Performance Supercapacitor. Small 2018, 14, 1702407. [CrossRef] [PubMed] 
17. He, Q.; Wang, Y.; Liu, X.X.; Blackwood, D.J.; Chen, J.S. One-pot synthesis of self-supported hierarchical urchin-like $\mathrm{Ni}_{3} \mathrm{~S}_{2}$ with ultrahigh areal pseudocapacitance. J. Mater. Chem. A 2018, 6, 22115-22122. [CrossRef]

18. Chen, W.; Xia, C.; Alshareef, H.N. One-Step Electrodeposited Nickel Cobalt Sulfide Nanosheet Arrays for High-Performance Asymmetric Supercapacitors. ACS Nano 2014, 8, 9531-9541. [CrossRef]

19. Yang, S.; Liu, Y.; Hao, Y.; Yang, X.; Goddard, W.A., III; Zhang, X.L.; Cao, B.Q. Oxygen-Vacancy Abundant Ultrafine $\mathrm{Co}_{3} \mathrm{O}_{4} /$ Graphene Composites for High-Rate Supercapacitor Electrodes. Adv. Energy Mater. 2018, $5,1702787$.

20. Shen, L.; Wang, J.; Xu, G.; Li, H.; Dou, H.; Zhang, X. NiCo $\mathrm{S}_{4}$ Nanosheets Grown on Nitrogen-Doped Carbon Foams as an Advanced Electrode for Supercapacitors. Adv. Energy Mater. 2015, 5, 1400977. [CrossRef]

21. Wen, P.; Fan, M.; Yang, D.; Wang, Y.; Cheng, H.; Wang, J. An asymmetric supercapacitor with ultrahigh energy density based on nickle cobalt sulfide nanocluster anchoring multi-wall carbon nanotubes hybrid. J. Power Sources 2016, 320, 28-36. [CrossRef]

22. He, W.; Liang, Z.; Ji, K.; Sun, Q.; Zhai, T.; Xu, X. Hierarchical Ni-Co-S@Ni-W-O core-shell nanosheet arrays on nickel foam for high-performance asymmetric supercapacitors. Nano Res. 2018, 11, 1415-1425. [CrossRef]

23. Wan, H.; Jiang, J.; Yu, J.; Xu, K.; Miao, L.; Zhang, L.; Chen, H.; Ruan, Y. $\mathrm{NiCo}_{2} \mathrm{~S}_{4}$ porous nanotubes synthesis via sacrificial templates: High-performance electrode materials of supercapacitors. CrystEngComm 2013, 15, 7649-7651. [CrossRef]

24. Chen, H.; Jiang, J.; Zhang, L.; Wan, H.; Qi, T.; Xia, D. Highly conductive $\mathrm{NiCo}_{2} \mathrm{~S}_{4}$ urchin-like nanostructures for high-rate pseudocapacitors. Nanoscale 2013, 5, 8879-8883. [CrossRef]

25. Xu, J.; Gao, L.; Cao, J.; Wang, W.; Chen, Z. Preparation and electrochemical capacitance of cobalt oxide (Co3O4) nanotubes as supercapacitor material. Electrochim. Acta 2010, 56, 732-736. [CrossRef]

26. Guan, B.; Yu, L.; Wang, X.; Song, S.; Lou, X.W. Formation of Onion-Like $\mathrm{NiCo}_{2} \mathrm{~S}_{4}$ Particles via Sequential Ion-Exchange for Hybrid Supercapacitors. Adv. Mater. 2017, 29, 1605051. [CrossRef]

27. Wang, G.; Zhang, L.; Zhang, J. A review of electrode materials for electrochemical supercapacitors. Chem. Soc. Rev. 2012, 41, 797-828. [CrossRef]

28. Li, Y.H.; Cao, L.; Qiao, L.; Zhou, M.; Yang, Y.; Xiao, P.; Zhang, Y. Ni-Co sulfide nanowires on nickel foam with ultrahigh capacitance for asymmetric supercapacitors. J. Mater. Chem. A 2014, 2, 6540-6548. [CrossRef]

29. Cai, D.; Xiao, S.; Wang, D.; Liu, B.; Wang, L.; Liu, Y.; Li, H.; Wang, Y.; Li, Q.; Wang, T. Morphology controlled synthesis of $\mathrm{NiCo}_{2} \mathrm{O}_{4}$ nanosheet array nanostructures on nickel foam and their application for pseudocapacitors. Electrochim. Acta 2014, 142, 118-124. [CrossRef]

30. Yan, M.L.; Yao, Y.D. Construction of a Hierarchical $\mathrm{NiCo}_{2} \mathrm{~S}_{4} @ P P y$ Core-Shell Heterostructure Nanotube Array on Ni Foam for a High-Performance Asymmetric Supercapacitor. ACS Appl. Mater. Interfaces 2016, 8, 24525-24535. [CrossRef]

31. Li, Y.; Zhang, Y.; Li, Y.; Wang, Z.; Fu, H.; Zhang, X. Unveiling the dynamic capacitive storage mechanism of $\mathrm{Co}_{3} \mathrm{O}_{4} @ \mathrm{NiCo}_{2} \mathrm{O}_{4}$ hybrid nanoelectrodes for supercapacitor applications. Electrochim. Acta 2014, 145, 177-184. [CrossRef]

32. Hao, P.; Tian, J.; Sang, Y.H.; Liu, H. 1D Ni-Co oxide and sulfide nanoarray/carbon aerogel hybrid nanostructures for asymmetric supercapacitors with high energy density and excellent cycling stability. Nanoscale 2016, 8, 16292-16301. [CrossRef]

(C) 2019 by the authors. Licensee MDPI, Basel, Switzerland. This article is an open access article distributed under the terms and conditions of the Creative Commons Attribution (CC BY) license (http://creativecommons.org/licenses/by/4.0/). 